\title{
ORIGINAL
}

\section{LA BRUCELOSIS COMO ENFERMEDAD PROFESIONAL: ESTUDIO DE UN BROTE DE TRANSMISION AEREA EN UN MATADERO}

\author{
M. ${ }^{a}$ Elena Rodríguez Valín (1), Anxela Pousa Ortega (1), Carmen Pons Sánchez (1), Alberto \\ Larrosa Montañés (2), Luisa P. Sánchez Serrano (1) y Ferrán Martínez Navarro (1).
}

(1) Centro Nacional de Epidemiología (Programa de Epidemiología Aplicada de Campo). Instituto de Salud Carlos III. Ministerio de Sanidad y Consumo.

(2) Diputación General de Aragón. Departamento de Sanidad, Consumo y Bienestar Social.

\section{RESUMEN}

Fundamento: La aparición de un número elevado de casos de brucelosis entre los trabajadores de un matadero en Zarago$\mathrm{za}$, en el que se sacrificaba ganado ovino procedente de las campañas de saneamiento ganadero, hizo iniciar una investigación para esclarecer el origen del brote.

Métodos: El estudio se estructuró en tres partes: 1) Descripción del brote; 2) Estudio de la estructura y actividad del matadero en cuanto al número de animales sacrificados, carga de trabajo del personal y riesgo por zona de trabajo y 3 ) Estudio de casos y controles no apareado.

Resultados: No hubo diferencias significativas entre las tasas de ataque por secciones de trabajo. El análisis del sacrificio reveló una variación concomitante entre las curvas de sacrificio de ovino de saneamiento y la epidémica. El estudio de casos y controles no reveló diferencias significativas para los siguientes factores de riesgo: trabajo en zona de riesgo, uso de medidas de protección y realización de cortes y heridas.

Conclusiones: El brote está relacionado con el sacrificio de ovino de saneamiento. El que no se observaran diferencias significativas por secciones o por trabajar en la zona de riesgo es compatible con una exposición generalizada. Tampoco se observaron diferencias entre los trabajadores respecto a la realización de cortes y heridas o el uso de medidas de protección, lo que da importancia a la transmisión aérea.

Palabras clave: Brote epidémico. Brucelosis. Brucelosis profesional. Transmisión aérea. Matadero.

Correspondencia:

M. ${ }^{a}$ Elena Rodríguez Valín.

Centro Nacional de Epidemiología

C/ Sinesio Delgado n. ${ }^{\circ} 6$

28029 Madrid

Correo electrónico: ervalin@isciii.es.

\section{ABSTRACT}

\section{Brucellosis as an Occupational Disease: Study of an Airborne Transmission Outbreak in a Slaughterhouse}

Background: The onset of a large number of cases of brucellosis among employees at a slaughterhouse in Zaragoza where sheep from livestock culling procedures were being slaughtered, led to an investigation being made for the purpose of shedding light on the cause of this outbreak.

Methods: This study was organized into three parts: 1) Description of the outbreak 2) Study of the slaughterhouse layout and activity as regards the number of animals slaughtered, the employee workload and the degree of risk involved in each working area and 3) Unmatched case-control study.

Results: No significant differences were found among the attack rates by the sections of the slaughterhouse where the employees in question worked. The slaughtering analysis revealed a concomitant fluctuation between the slaughter of culled sheep and the epidemic. The case-control study revealed no significant differences for the risk factors involving working in a risk area, the use of protective measures and cuts/wounds.

Conclusions: This outbreak is related to the slaughter of culled sheep. The fact that no significant difference were found by sections or due to working in a risk area is compatible with an overall explanation. Nor were any differences among the employees found to exist with regard to cuts/wounds or the use of protective measures, which focuses importance on airborne spread.

Keywords: Outbreak. Brucellosis. Occupational. Airborne spread. Slaughterhouse. 


\section{INTRODUCCIÓN}

La brucelosis es una zoonosis producida por distintas especies del Género Brucella, cuyas fuentes de infección y organismo responsable varían en función de la zona geográfica $^{1}$. En España, al igual que en el resto de países de la Cuenca Mediterránea, es una enfermedad muy ligada al ganado ovino y caprino; la vía directa es el mecanismo de contagio más frecuente en algunas regiones españolas, lo que es indicativo de su perfil ocupacional $^{2,3}$. Fue declarada enfermedad profesional en $1978^{4}$.

El control en los animales se realiza a través del Programa Nacional de Erradicación de la brucelosis ovina y caprina, por medio de las campañas de saneamiento ganadero, que permite el sacrificio de los animales positivos a las pruebas de brucelosis en mataderos debidamente autorizados 5 . De los 15 brotes declarados al Centro Nacional de Epidemiología desde 1989, 12 están relacionados con el sacrificio de ganado ovino procedente de campañas de saneamiento ganadero (Fuente: Centro Nacional de Epidemiología).
Entre el 26 de Diciembre de 1998 y el 4 de Mayo de 1999 se produjeron un total de 28 casos de brucelosis en un matadero de Zaragoza, de los cuales 24 se dieron en trabajadores del matadero del turno de mañana y 4 eran en trabajadores de otras empresas pero con relación laboral con el mismo matadero. Éste está autorizado desde 1991 para el sacrificio de animales procedentes de campañas de saneamiento ganadero. Las especies sacrificadas son: vacuno, porcino, equino, y sobre todo ovino y caprino. El ovino positivo a brucelosis se sacrifica mayoritariamente, junto con el caprino, por los trabajadores del turno de mañana, y en el caso de que queden animales al finalizar la jornada laboral, estos son sacrificados por los trabajadores del turno de tarde.

En este matadero hasta 1991 se producían casos esporádicos de brucelosis, y desde 1991 se comenzaron a producir brotes con un pequeño número de casos; el más relevante fue el producido en 1997 con 9 casos (figura 1).

Figura 1

Evolución de los casos de brucelosis entre los trabajadores del matadero (1981-1998)

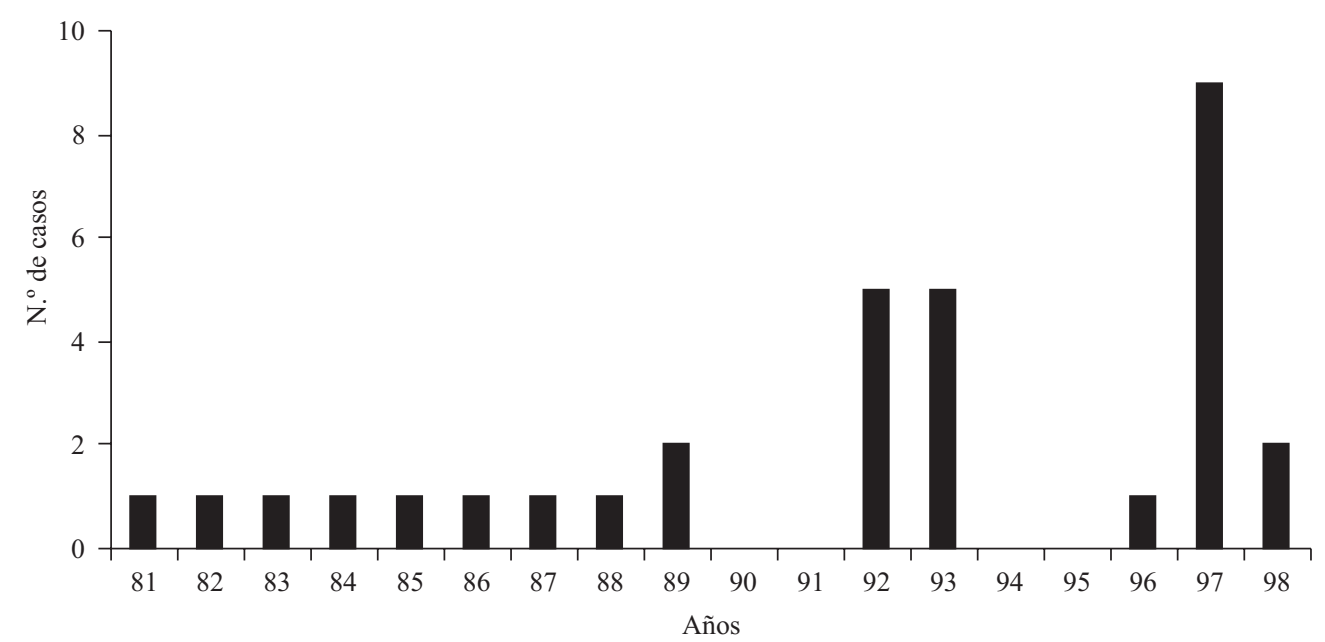


Respecto al sacrificio de ganado ovino de saneamiento, éste ha ido aumentando paulatinamente con el tiempo, pasando de 2.010 cabezas sacrificadas en 1996 a 13.206 cabezas en 1998. En cuanto al ganado caprino de saneamiento, es habitual que éste venga mezclado con el ovino, oscilando la relación caprino/ovino entre $1 / 20$ y $1 / 150$. El sacrificio de ganado vacuno positivo a brucelosis es ocasional; entre octubre del 98 y febrero del 99 sólo se sacrificaron 8 cabezas.

Teniendo en cuenta esta información, parecía lo más probable relacionar el brote con el sacrificio de ganado ovino procedente de las campañas de saneamiento. Los objetivos de este trabajo fueron en primer lugar realizar el estudio descriptivo del brote respecto a los parámetros de tiempo, lugar y persona; y posteriormente tratar de determinar el origen del brote y aquellos factores de riesgo que habían contribuido a su aparición.

\section{MATERIAL Y MÉTODOS}

1. Estudio descriptivo del brote en cuanto a las variables de tiempo, lugar y persona.

2. Estudio de estructura y actividad del matadero, para lo cual se realizaron varias visitas con el fin de conocer las condiciones locales y otros factores que puedan influir en las condiciones ambientales:

- Análisis de riesgos de contagio de brucelosis en las distintas secciones según el contacto de los trabajadores con aerosoles, sangre, tejidos, vísceras o fluidos de los animales, tras lo cual se elaboró un mapa de riesgos ${ }^{6}$.

- Evolución del sacrificio del ganado ovino positivo a Brucella desde 1996; especialmente durante el brote y en los meses previos compatibles con el período de incubación.

- Valoración de la carga de trabajo del personal, desde Octubre de 1998 hasta Febrero de 1999, para lo cual se calculó el tiempo empleado diariamente en el faenado de cada especie y se calculó el promedio de horas diarias por empleado en cada turno y cada mes del período.

3. Estudio de casos y controles no apareado a fin de valorar la exposición a factores de riesgo. La población de referencia constaba de 122 trabajadores; se pudo obtener información del $86,8 \%$ de éstos (106). Para este estudio se establecieron las siguientes definiciones:

- Caso: persona que había trabajado en el matadero entre el 15 de Noviembre de 1998 y el 12 de Marzo de 1999, que presentaba algunos de los síntomas siguientes: fiebre, sudoración, astenia, artralgias y orquitis; y con unos títulos de seroaglutinación en tubo mayores de 1/80 acompañados de un test de Coombs mayor de 1/320 y/o aislamiento de brucela por hemocultivo.

- Control: persona que había trabajado en el matadero entre el 15 de Noviembre de 1998 y el 12 de Marzo de 1999, sin sintomatología compatible con brucelosis, con aglutinación y test de Coombs negativos.

Teniendo en cuenta estos criterios, se consideraron 28 casos y 78 controles.

Las pruebas de laboratorio realizadas a los trabajadores consistieron en: test Rosa de Bengala, seroaglutinación en tubo (SAT), test de Coombs, y en algunos de ellos hemocultivo. La identificación de la especie y biovariedad de las cepas de Brucella sp se realizó en el Laboratorio de Microbiología de la Universidad de Valladolid (Facultad de Medicina).

Para la recogida de la información necesaria para este estudio se diseñó una encuesta en la que se incluían las siguientes variables:

- variables personales: edad, sexo, antecedentes de brucelosis. 
- variables de exposición no laboral: consumo de productos lácteos no higienizados y contacto con animales distintos a los del matadero.

- variables de exposición laboral: antigüedad en el matadero, contacto con ganado ovino procedente de campañas de saneamiento, uso de medidas de protección individual, cortes y heridas durante el trabajo, realizar funciones de limpieza, tareas desempeñadas, tiempo empleado en el sacrificio de ovino de saneamiento y otras especies, y condiciones de humedad y temperatura en su trabajo.

Las variables cuantitativas relativas a los trabajadores (edad, antigüedad laboral) se caracterizaron por su media y desviación estándar. Las variables cualitativas se caracterizaron mediante el cálculo de los porcentajes para cada una de las categorías con sus Intervalos de Confianza al 95\% ( $\left.\mathrm{IC}_{95 \%}\right)$. Para el análisis estadístico de los datos se empleó el programa Epiinfo V 6.0. En el estudio de casos y controles, la asociación de la variable dependiente (caso de brucelosis) con las diferentes variables de exposición se realizó mediante el cálculo de la Odds Ratio con $\mathrm{su} \mathrm{IC}_{95 \%}$, considerando significativos valores de $\mathrm{p}<0,05$.
Otras fuentes de información, además de la encuesta epidemiológica fueron las siguientes: partes de declaración de la Mutua Laboral (datos de las pruebas diagnósticas), registros del Servicio médico de empresa (fechas de baja y brucelosis anteriores), estadísticas de sacrificio de los Servicios de Inspección Veterinaria del matadero (número de animales sacrificados de cada especie por día y turno); y entrevistas a los trabajadores, veterinarios, comité de empresa y jefe de producción (estudio de la estructura y actividad del matadero), así como los resultados de la visita al matadero.

\section{RESULTADOS}

Los resultados del estudio descriptivo respecto al tiempo muestran una curva epidémica de distribución bimodal (figura 2), con una primera onda que se corresponde con las tres primeras semanas del brote y una segunda onda donde se acumulan la mayor parte de los casos (semanas epidemiológicas de la 4 a la 8). Los parámetros de dinámica de la curva indican una desviación hacia la derecha y una difusión lenta de la enfermedad (retardo medio ponderado 7,8 semanas, desviación estándar 4,2, curtosis 2,2, asimetría - 0,2 y coeficiente de difusión 0,02).

Figura 2

Curva epidémica por semanas epidemiológicas

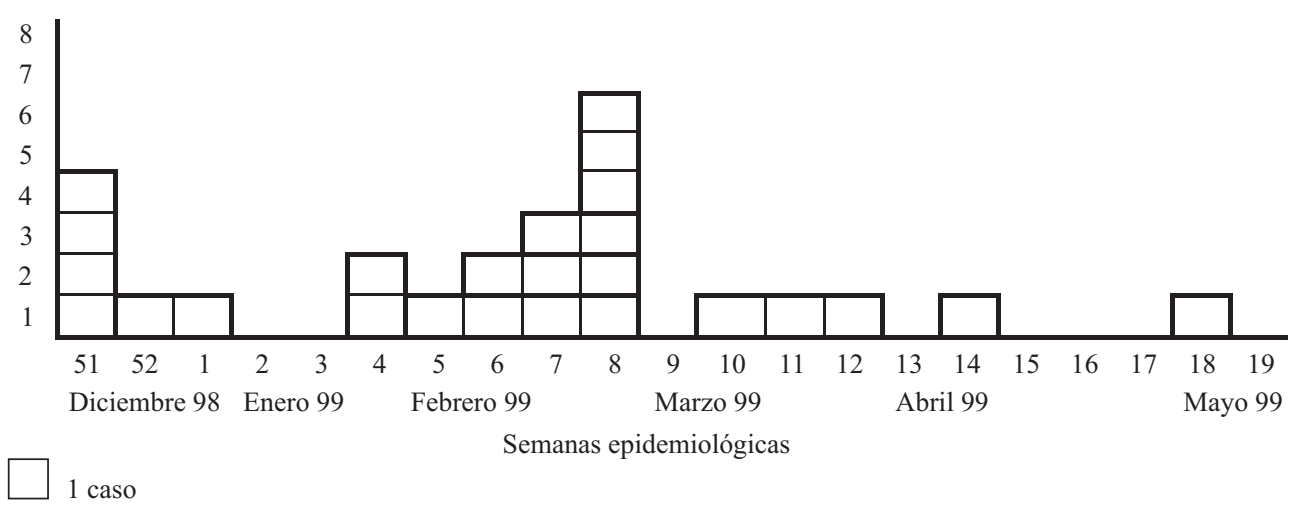


Respecto al cuadro clínico de las personas incluidas como casos, $20(71,4 \%)$ presentaron fiebre, $20(71,4 \%)$ sudoraciones, 21 (75\%) astenia y 25 artralgias (89,3\%). Dos de ellos $(7,1 \%)$ presentaron orquitis y dos $(7,1 \%)$ artritis. Todos ellos fueron analizados en el laboratorio mediante las pruebas del Rosa de Bengala, aglutinaciones antibrucela y test de Coombs. En un hemocultivo se aisló $B$. melitensis biotipo 1 .

Las características personales de los casos fueron: 27 (96,4\%) hombres, edad media 38,8 años (desviación estándar=9,1), antigüedad laboral media 11,9 años (desviación estándar $=6,5$ ) y sólo uno tenía antecedentes de brucelosis.

En cuanto a la distribución de los casos por tipo de actividad se observa que todas las secciones están afectadas y (aunque las tasas de ataque más altas se encuentran en sacrificio, faenado y despojos) que no hay diferencias significativas entre las tasas de ataque de unas secciones y otras (tabla 1).

Como resultado de la visita al matadero se elaboró un mapa de riesgos ${ }^{6}$, en el que se establecieron las siguientes zonas:

- Zona I (riesgo elevado), en la que incluimos las secciones de sacrificio, faenado y despojos. En estas fases el riesgo de contagio sería elevado, por la exposición a aerosoles y por el contacto con fluidos y/o tejidos infectados.

—Zona II (riesgo medio), que comprende las secciones de cámaras (oreo y conservación) y la sala de ventas. En estas secciones la exposición a Brucella es escasa, ya que las canales están limpias.

—Zona III (riesgo bajo), en la que está incluido el personal de servicios (limpieza, mantenimiento, albañiles, soldadores), el de corrales y el de Sanidad (Servicios veterinarios). En estas actividades el riesgo de contagio es bajo ya que la exposición a animales o sus partes es breve o nula.

Los resultados del análisis de la carga de trabajo se muestran en la tabla 2; este análisis se realizó con el fin de comparar el diferente volumen de trabajo entre los turnos de mañana y de tarde, y el tiempo medio que cada empleado tendría que pasar en la cadena de sacrificio. Las cifras no se deben de considerar de manera absoluta, hay que tener en cuenta que se suelen sacrificar simultáneamente varias especies y que los operarios se van turnando por las distintas lineas de sacrificio, por lo que se solapan los tiempos (una jornada normal de trabajo es de 8-10 horas). Observamos que para el total del período estudiado (Octubre - 98 a Febrero - 99) el turno de mañana tiene una carga de trabajo 4 veces superior al de tarde.

Tabla 1

Distribución de los casos de brucelosis por secciones de trabajo

\begin{tabular}{|lccccccc|}
\hline \multicolumn{1}{|c}{ Sección } & casos & no casos & TA (\%) & RR & & IC 95\% & $p$ \\
\hline Sacrificio & 1 & 2 & 33,3 & 3,00 & $0,26-34,68$ & 0,45 \\
Faenado & 13 & 32 & 28,8 & 2,60 & $0,39-17,5$ & 0,25 \\
Despojos & 5 & 11 & 31,3 & 3,81 & $0,39-20,5$ & 0,26 \\
Cámaras & 2 & 7 & 22,2 & 2,00 & $0,22-18,3$ & 0,50 \\
Limpieza & 1 & 8 & 11,1 & 1 & & \\
Mantenimiento & 2 & 6 & 25,0 & 2,25 & $0,25-20,4$ & 0,45 \\
Empresas Externas & 3 & 6 & 33,3 & 3,00 & $0,38-23,7$ & 0,28 \\
Servicios C. Sanidad & 1 & 7 & 12,5 & 1,13 & $0,08-15,2$ & 0,73 \\
\hline
\end{tabular}

* TA: tasa de ataque, RR: riesgo relativo, IC: intervalo de confianza. 
Tabla 2

Análisis de la carga de trabajo del personal *. Comparación entre los turnos

\begin{tabular}{|c|c|c|}
\hline Meses & Turno mañana & Turno tarde \\
\hline Octubre-98 & 20,35 & 5,33 \\
\hline Noviembre-98 & 23,64 & 4,50 \\
\hline Diciembre-98 & 24,97 & 7,30 \\
\hline Enero-99 & 14,51 & 4,80 \\
\hline Febrero-99 & 15,26 & 5,31 \\
\hline Media período & 20,38 & 5,09 \\
\hline
\end{tabular}

* Carga de trabajo en horas /empleado/día. El número de animales sacrificados diariamente se multiplicó por el tiempo que permanece cada uno en la cadena de sacrificio (desde que son sacrificados hasta que entran en cámaras), este tiempo varía en función de la especie. Posteriormente esta cifra se dividió entre el número total de empleados que intervienen en la cadena.

Se estudió también la evolución del sacrificio de ovino de saneamiento en el matadero (tabla 3). Si se comparan las cifras de 1996 con las de 1998 vemos que este sacrificio aumentó 6 veces (de 2.010 cabezas en 1996 a 13.206 en 1998), aunque de forma gradual en el tiempo. Respecto al período del brote, en la figura 3 se aprecia una variación concomitante de las curvas de sacrificio de ovino de saneamiento y la epidémica. En cuanto al sacrificio de otras especies, el de vacuno se mantuvo constante a lo largo de este período, y el de ovino y caprino me- nor (corderos y cabritos) tuvo su máximo en diciembre del 98 a consecuencia de la campaña de navidad, coincidiendo con el mínimo o nulo sacrificio de ganado de saneamiento.

En el estudio de casos y controles se consideró una población a riesgo de 122 personas, de las que fueron encuestadas 106 $(86,8 \%)$. Los 16 trabajadores restantes no pudieron ser encuestados por motivos varios como encontrarse fuera o negarse a colaborar.

Tabla 3

Evolución del sacrificio de ganado ovino de saneamiento en el matadero * (1996-1998)

\begin{tabular}{|lrrr|}
\hline & Meses & 1996 & 1997 \\
\hline Enero & 138 & 146 & 123 \\
Febrero & 2 & 430 & 336 \\
Marzo & 73 & 1.129 & 643 \\
Abril & 238 & 383 & 1.353 \\
Mayo & 127 & 763 & 793 \\
Junio & 582 & 363 & 827 \\
Julio & 199 & 1.357 & 2.349 \\
Agosto & 83 & 281 & 2.225 \\
Septiembre & 16 & 670 & 1.229 \\
Octubre & 227 & 828 & 1.541 \\
Noviembre & 126 & 374 & 1.041 \\
Diciembre & 199 & 36 & 746 \\
Total & & $\mathbf{6 . 7 6 0}$ & $\mathbf{1 3 . 2 0 6}$ \\
\hline
\end{tabular}

* número de cabezas. 
Figura 3

Relación entre el sacrificio de ganado ovino de saneamiento en el matadero y la aparición de casos

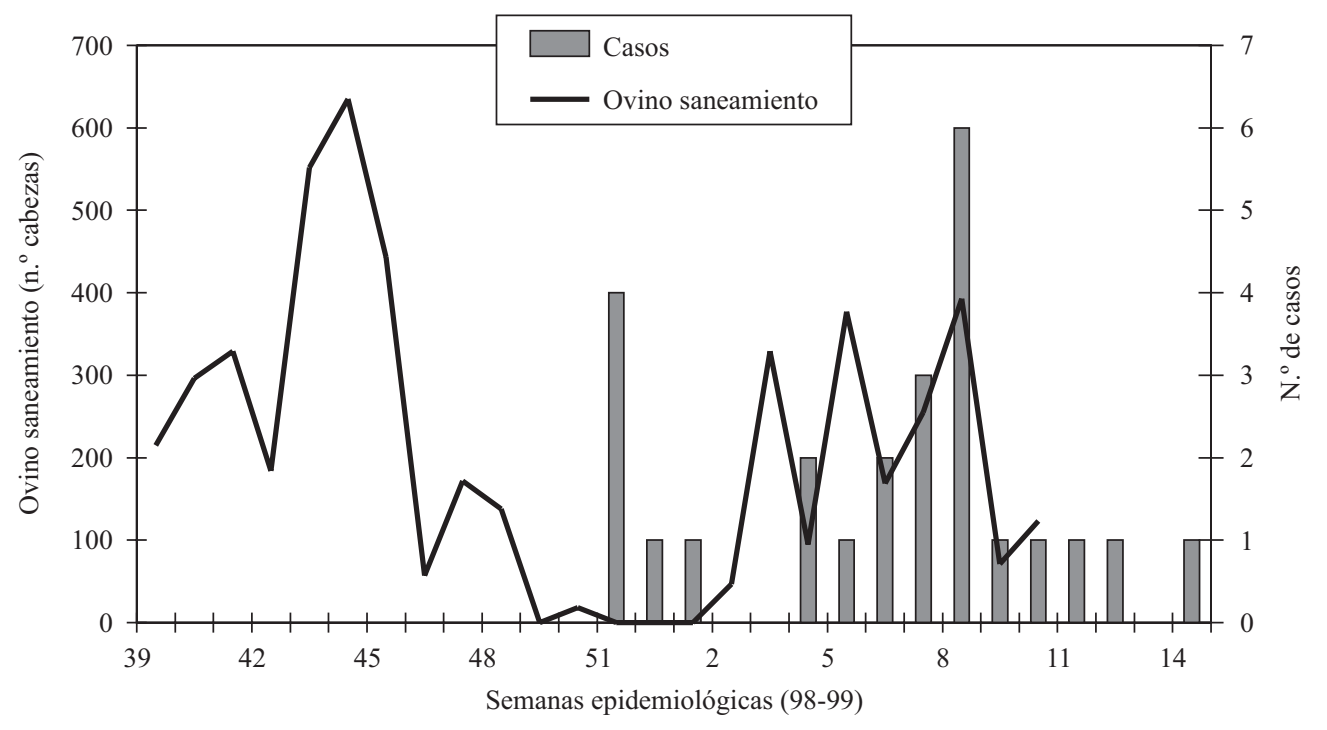

Las características personales de los controles fueron muy similares a las de los casos: el 97,4\% (76) fueron hombres, la edad media fue de 42,8 años (desviación estándar $=9,5)$ y la antigüedad laboral media de 12,7 años (desviación estándar $=5,6$ ). El $27 \%$ (21) de ellos tenían antecedentes de brucelosis.

Los factores de riesgo estudiados fueron: consumo de productos lácteos no higienizados, contacto con animales fuera del matadero, trabajar de forma habitual o esporádica en la zona I (zona de riesgo elevado de contagio), haber padecido brucelosis, haber sufrido heridas y cortes durante el trabajo y usar medidas de protección individual. Los resul- tados más relevantes se muestran en la tabla 4. No se observaron diferencias significativas entre los trabajadores según realizaran su trabajo de forma habitual o esporádica en la zona I (OR de 2,01 con $\mathrm{IC}_{95 \%}$ : 0,74-5,51). Tampoco se observaron diferencias significativas entre enfermos y no enfermos según la producción de heridas y cortes durante el trabajo (OR de 1,47 con $\mathrm{IC}_{95 \%}$ : 0,56-3,85) ni por el uso de medidas de protección (OR de 0,88 con $\left.\mathrm{IC}_{95 \%}: 0,31-2,50\right)$.

Al estudiar los antecedentes de enfermedad en los trabajadores se encontró que aquellos que habían padecido brucelosis tenían un menor riesgo de contraer la enfermedad (OR de 0,1 con $\mathrm{IC}_{95 \%}, 0,00-0,77$ ). 
Tabla 4

Estudio de casos y controles. Riesgo de enfermar en los trabajadores según las diferentes variables de exposición estudiadas

\begin{tabular}{|c|c|c|c|c|c|}
\hline FACTOR DE RIESGO & & Casos & $\begin{array}{c}\text { Contro- } \\
\text { les }\end{array}$ & OR (IC 95\%) & $p$ \\
\hline \multicolumn{6}{|l|}{ Trabajo habitual en zona riesgo } \\
\hline zona I & & 19 & 40 & 2,01 & 0,13 \\
\hline zonas II y III & & 9 & 38 & $(0,74-5,51)$ & \\
\hline \multicolumn{6}{|l|}{ Trabajo esporádico en zona riesgo } \\
\hline zona I & & 28 & 68 & 4,62 & 0,11 \\
\hline zona II y III & & 0 & 10 & $(0,52-100,13)$ & \\
\hline \multicolumn{6}{|l|}{ Heridas y cortes } \\
\hline \multirow{2}{*}{ general } & sí & 14 & 30 & 1,47 & 0,39 \\
\hline & no & 14 & 44 & $(0,56-3,85)$ & \\
\hline \multirow{2}{*}{ herramientas sucias } & sí & 13 & 22 & 1,77 & 0,63 \\
\hline & no & 1 & 3 & $(0,14-49,29)$ & \\
\hline \multicolumn{6}{|l|}{ Uso medidas de protección } \\
\hline \multirow{2}{*}{ general } & sí & 19 & 55 & 0,88 & 0,79 \\
\hline & no & 9 & 23 & $(0,31-2,50)$ & \\
\hline \multirow{2}{*}{ cutánea (guantes) } & sí & 19 & 55 & 0,88 & 0,79 \\
\hline & no & 9 & 23 & $(0,31-2,50)$ & \\
\hline \multirow{2}{*}{ aérea (mascarilla) } & sí & 18 & 48 & 1,13 & 0,79 \\
\hline & no & 10 & 30 & $(0,42-3,07)$ & \\
\hline \multirow{2}{*}{ ocular (gafas) } & sí & 16 & 39 & 1,33 & 0,66 \\
\hline & no & 12 & 39 & $(0,50-3,51)$ & \\
\hline \multirow{2}{*}{ Antecedentes de brucelosis } & sí & 1 & 21 & 0,11 & 0,009 \\
\hline & no & 27 & 57 & $(0,00-0,77)$ & \\
\hline
\end{tabular}

\section{DISCUSIÓN}

De la observación de la figura 3 se deduce una relación entre el sacrificio de ovino de saneamiento positivo a Brucella y la aparición de casos. Entre 6 y 8 semanas antes del comienzo del brote se sacrifica un elevado número de ovejas de saneamiento, lo que es compatible con el período de incubación de la brucelosis ${ }^{7}$. Las semanas en las que no se realiza sacrificio no van seguidas de la aparición de casos, y éstos se vuelven a producir al reanudarse el sacrificio. El aislamiento de $B$. melitensis en uno de los casos hace sospechar del ovino de saneamiento como especie responsable del brote.
La infección del ganado bovino por $B$. melitensis se ha descrito en la literatura, y se suele producir cuando el ganado comparte instalaciones o pastos con ovinos o caprinos infectados ${ }^{8}$. Esto representa un problema emergente en algunos países mediterráneos no europeos como Israel, $\mathrm{Ku}-$ wait o Arabia Saudí ${ }^{9}$. No obstante, el escaso número de bovinos positivos a Brucella en este matadero no nos hace posible sospechar de esta especie como causante del brote. Resulta más plausible relacionarlo con el sacrificio de ovino o caprino infectado ya que estas especies son las afectadas habitualmente por B. melitensis. Aunque el caprino y ovino positivos a Brucella van 
mezclados en el momento del sacrificio, el mayor volumen del segundo respecto del primero nos hace inclinarnos hacia el ganado ovino de saneamiento como especie responsable del brote.

El aumento del sacrificio de ovino de saneamiento es un factor que contribuye a aumentar el riesgo de infección ${ }^{10,11,12}$. En este matadero dicho sacrificio se multiplicó por 6 en los dos últimos años. Este hecho es especialmente relevante en los trabajadores del turno de mañana, lo que unido a su carga de trabajo 4 veces superior podría contribuir a que dichos trabajadores tuvieran mayor riesgo de adquirir brucelosis que los del turno de tarde.

La importancia de la vía de transmisión aérea y el aislamiento de Brucella en el ambiente del matadero ha sido reseñada en varios estudios ${ }^{6,13,14}$. Para valorar este aspecto se realizó un análisis de riesgos y una clasificación de las actividades en tres zonas.

Los resultados de este estudio apoyan la hipótesis de la transmisión aérea. Ello se debe a:

1. No hay diferencias según el riesgo entre las zonas de trabajo, lo que se demuestra por el estudio de las tasas de ataque por secciones y por los resultados del estudio de casos y controles. Sin embargo, aunque el análisis estadístico no evidencie diferencias significativas entre los trabajadores habituales de la zona I (zona de mayor riesgo) y del resto, lo cierto es que entre los trabajadores habituales de esta zona se produjeron 19 casos (40 controles) frente a 9 casos (y 38 controles) entre los que no trabajan habitualmente en ella. También hay que señalar que estudiando la circulación del personal por el matadero se observó que la zona I es zona de paso obligado hacia los aseos y vestuarios de los trabajadores, y que además el personal externo había realizado trabajos en dicha zona.
2. No se observaron diferencias significativas entre aquellos trabajadores que realizaban operaciones de riesgo, con la consiguiente producción de cortes y heridas, frente a los que no las realizaban.

3. Tampoco se observaron diferencias entre casos y no casos respecto a la utilización de medidas de protección personal en general, ni en particular respecto a las de protección cutánea, ocular o respiratoria.

Teniendo en cuenta estos tres hechos, y que los trabajadores de mataderos se encuentran expuestos simultáneamente a las vías de contagio cutánea y aérea ${ }^{6,11}$, se puede sospechar que la vía de transmisión predominante en el brote ha sido la aérea y que las medidas de protección personal han tenido poca eficacia ${ }^{15}$.

Hay estudios que señalan que trabajadores de mataderos previamente infectados por Brucella adquieren una inmunidad relativa que les proporcionaría una protección del $92 \%$ frente a la reinfección ${ }^{14}$. Nuestros resultados parecen concordar con estas afirmaciones. De los 28 casos, sólo uno había padecido brucelosis, mientras que de los 78 controles, 21 habían padecido la enfermedad en algún momento de su vida laboral. No obstante, al no estar plenamente demostrada la existencia de inmunidad en la brucelosis, pensamos que estos resultados deben considerarse con prudencia y que sería necesario realizar observaciones en un número mayor de casos.

En este estudio no se ha podido disponer de información acerca de seroconversión ni se han realizado otras pruebas diagnósticas más precisas como ELISA, por lo que para la definición de caso se han tenido que establecer criterios de inclusión basados en la presencia o no de síntomas, y en los resultados de las pruebas de seroaglutinación y test de Coombs. Aunque el punto de corte recomendado en la prueba de seroaglutinación es de $1 / 160^{7}$, pensamos que la presencia de síntomas, junto con unos títulos de seroa- 
glutinación de 1/80 más un test de Coombs de 1/320 son criterios de inclusión adecuados para los casos, máxime teniendo en cuenta que todos los trabajadores son sometidos a test rutinarios anuales en el matadero. Los 4 casos que tenían unos títulos de seroaglutinación de 1/80 presentaban una sintomatología acusada, y el resto tenían además títulos superiores a 1/160.

Respecto a la sintomatología, en este estudio hemos considerado casos a 3 trabajadores que manifestaron no presentar síntomas (sus títulos en la prueba de seroaglutinación eran superiores a 1/160); para ello nos basamos en sus antecedentes: una de ellas había estado de baja por amigdalitis en el mes de Enero-99, y las tres habían dado negativas en las pruebas rutinarias realizadas a los trabajadores el año anterior. Aunque se ha descrito la existencia de brucelosis subclínica $^{16}$; teniendo en cuenta lo anterior, lo inespecífico de la sintomatología de la brucelosis, y el gran esfuerzo físico y la elevada carga de trabajo a que se ven sometidos los trabajadores de mataderos, pensamos que es más probable que estos síntomas pasaran desapercibidos que el que no existiesen. No obstante, al ser un número poco representativo (sólo 3 trabajadores), los resultados del análisis estadístico no varían tanto si se incluyen en el grupo de casos como si se incluyen en el de controles.

Finalmente, se podría discutir si el brote coincide con alguna situación accidental, cambio en las condiciones de trabajo, etc. Tenemos constancia que en este matadero se realizaron obras de cambio de suelos, pintura, sustitución del silo de decomisos, etc, entre los meses de Noviembre de 1998 y Enero de 1999. No obstante, no se ha podido demostrar que estas obras tuvieran repercusión en la ocurrencia del brote, ya que se realizaban en fines de semana y festivos, o en momentos en los que no existía actividad en el matadero.

Como principales conclusiones de este estudio se pueden señalar las siguientes: 1 .
El brote está muy posiblemente relacionado con el sacrificio de ovino de saneamiento, al ser esta especie la de mayor volumen e importancia entre las positivas a Brucella sacrificadas en el matadero. 2. La vía de transmisión más probable fue la aérea y la difusión generalizada, 3. La vía de transmisión cutánea no juega un papel destacado, y 4. Las medidas de protección personal utilizadas no parecen ser eficaces contra la infección.

Finalmente, para prevenir la aparición de brucelosis en los trabajadores de mataderos, se señalan las siguientes recomendaciones: 1. Aunque en este trabajo no se ha demostrado la eficacia de las medidas de protección personal, en otros brotes éstas sí han demostrado su utilidad, por lo que no se puede dejar de recomendar su uso ${ }^{11,16}$. Nosotros recomendamos mejorar dichas medidas, especialmente las respiratorias, utilizando respiradores de partículas similares a los empleados para prevenir la transmisión de la tuberculosis ${ }^{17}, 2$. Espaciar el número de animales positivos sacrificados diariamente ${ }^{10,13}$, y 3 . Control de la cabaña ganadera, especialmente de ovino - caprino, lo que incluiría el incentivar en los ganaderos otras medidas para eliminar el ganado enfermo (enterramiento, incineración, etc.) en lugar del sacrificio en el matadero ${ }^{16}$.

En este brote en particular, y tras la realización de este estudio, se señaló que sería conveniente realizar un análisis ambiental del matadero, así como un estudio general de los brotes de brucelosis en los mataderos españoles con objeto de determinar la importancia de salud laboral de este problema.

\section{AGRADECIMIENTOS}

Los autores agradecen a los Servicios de Epidemiología y de Higiene Alimentaria de la Diputación General de Aragón la información y ayuda facilitada para el estudio del brote, así como la colaboración prestada por los trabajadores del matadero para la inves- 
tigación (Dirección, Jefe de Producción, Comité de Empresa, Servicio Médico de Empresa y Servicios Veterinarios Oficiales). También agradecemos las aportaciones de Marta Zimmerman y de Jerónimo Maqueda, del Instituto Nacional de Seguridad e Higiene en el Trabajo.

\section{BIBLIOGRAFÍA}

1. Benenson Abram S. El control de las enfermedades transmisibles en el hombre. 16. ${ }^{a}$ Ed. Washington: OPS 1997. Publicación Científica núm 564.

2. Serra J, Pujol R, Godoy P. Estudio seroepidemiológico de brucelosis en un área rural endémica. Enferm Infecc Microbiol Clin 2000; 18: 74-8.

3. Serra Alvarez J, Godoy García P. Incidencia, etiología y epidemiología de la brucelosis en un área rural de la provincia de Lérida. Rev Esp Salud Pública 2000; 74: 45-53.

4. Boletín Oficial del Estado. Real Decreto 1995/1978 que aprobó el cuadro de enfermedades profesionales en el sistema de la Seguridad Social. BOE núm 203, 25/8/1978.

5. Boletín Oficial del Estado. Real Decreto 2611/1996 que regula los Programas Nacionales de erradicación de enfermedades de los animales. BOE núm 307, 21/12/ 1996.

6. Kaufmann AF, Fox MD, Boyce JM, Anderson DC, Potter ME, Martone WJ et al. Airborne spread of brucellosis. Ann N Y Acad Sci 1980; 353: 105-114.

7. Young EJ. Brucella species. En: Mandell GL, Bennett JE, Dolin R, eds. Mandell, Douglas, and Bennett's Principles and Practice of Infectious Diseases. 5. ${ }^{\mathrm{a}}$ ed. Londres: Churchill Livingstone; 2000. p.2386-93.
8. Acha PN, Szyfres B. Zoonosis y enfermedades transmisibles comunes al hombre y a los animales, 2. ${ }^{\mathrm{a}}$ ed. Organización Panamericana de la Salud. Publicación Científica n. ${ }^{\circ}$ 503. 1986. p. 14-36.

9. Corbel MJ. Brucellosis: an Overview. Emerg Infect Dis 1997; 3 (2): 213-221.

10. Davos DE, Cargill CF, Kyrkou MR, Jamieson JA, Rich GE. Outbreak of brucellosis at a South-Australian abattoir. Med J Aust 1981; 2 : 657-660.

11. Alleyne BC, Orford RR, Lacey BA, White FMM. Rate of slaughter may increase risk of human brucellosis in a meat-packing plant. J Occup Med 1986; 28: 445-450.

12. Luna A., Rodríguez de Cepeda A., Suárez T. Análisis de un brote epidémico de brucelosis en trabajadores de un matadero. Rev Esp Salud Pública $1998 ; 72: 137-146$.

13. White PC, Baker EF, Roth AJ, Williams WJ, Stephens TS. Brucellosis in a Virginia meat-packing plant. Arch Environ Health 1974; 28 263-271.

14. Buchanan TM, Hendriks, SL, Patton CM, Feldman RA. Brucellosis in the United States, 1960-1972 (Part III). Medicine 1974; 53 427-439.

15. Schnurrenberger PR, Martin RJ, Wactor PR, Jelly GG. Brucellosis in an Illinois abattoir. Arch Environ Health 1972; 24: 337-341.

16. Trout D, Gómez TM, Bernard BP, Mueller CA, Smith CG, Hunter L et al. Outbreak of brucellosis in a United States pork packing plant. J Occup Environ Med 1995; 37: 697-703.

17. Centers for Disease Control and Prevention. Guidelines for Preventing the Transmission of $\mathrm{Myco}$ bacterium tuberculosis in Health-Care Facilities. MMWR 1994; 43 (RR-13). 